\title{
Percepciones sobre riesgos ocupacionales en el trabajo con animales y motivaciones de los estudiantes para ingresar a la carrera de veterinaria
}

\author{
Tarabla $\mathrm{HD}^{1}$ \\ ${ }^{1}$ Facultad de Ciencias Veterinarias, Universidad Nacional del Litoral (FCV-UNL), Argentina. \\ * Correspondencia: Héctor Tarabla, FCV-UNL, RP Kreder 2805, 3080 Esperanza, Santa Fe, Argentina. \\ E-mail: epidemiologia21@gmail.com
}

Recibido: 15 Marzo 2020. Aceptado: 25 Mayo 2021. Disponible en línea: 19 Julio 2021

Editor: P. Beldomenico

RESUMEN. Se desarrolló un estudio transversal en 130 ingresantes a la Faculta de Ciencias Veterinarias, Universidad Nacional del Litoral para describir las motivaciones para estudiar veterinaria y las percepciones sobre el riesgo de sufrir enfermedades profesionales y accidentes ocupacionales. El análisis estadístico incluyó $x 2$ y correlación de Spearman. La motivación por la medicina fue la más prevalente, registrándose un mayor interés por los bovinos, caninos, felinos y fauna silvestre. Los varones mostraron preferencia por los bovinos $(P=0,005)$ y las mujeres por los caninos y felinos $(P<0,001)$. La motivación por la actividad agropecuaria y los espacios abiertos no estuvieron significativamente asociadas $(P=0,598)$. La primera estuvo relacionada con los bovinos $(P<0,001)$, ovinos $(P=0,003)$ y porcinos $(P=0,050)$, mientras que los últimos sólo con la fauna silvestre $(P<0,053)$. Las zoonosis más mencionadas fueron rabia, tuberculosis y brucelosis. Los estudiantes percibieron el riesgo de enfermedades como más alto en el trabajo con porcinos y bovinos, mientras que el de lesiones lo fue con equinos. Las respuestas "No sabe/no contesta" fueron frecuentes en el reconocimiento de algunas zoonosis y en las percepciones de riesgos de accidentes y enfermedades. Estos resultados reafirman la necesidad de un contacto precoz de los estudiantes de veterinaria con temas relacionados con la salud ocupacional.

SUMMARY. Occupational risk perceptions in working with animals and motivations to study veterinary medicine in freshmen. A cross-sectional study was carried out in 130 freshmen at the School of Veterinary Science, Litoral National University to describe the motivations for studying veterinary medicine and their perceptions about the risk of suffering occupational diseases or occupational accidents. Statistical analysis included $\chi 2$ and Spearman's correlation coefficient. Motivations were high for medical sciences, bovines, canines, felines and wildlife. Males showed preference for bovines $(P=0.005)$, while females preferred canines and felines $(P<0.001)$. Interests in agricultural activity and open spaces were not significantly associated $(P=0.598)$. The former was related to cattle $(P<0.001)$, sheep $(P=0.003)$ and pigs $(P=0.050)$, while the latter only to wildlife $(P<0.053)$. The most mentioned zoonosis were rabies, tuberculosis and brucellosis. The perception of the risk of zoonotic diseases was higher in pigs and cattle, while that of lesions was higher in horses. The "Don't know / No response" answers were frequent in the recognition of some zoonoses and the perceptions of the risks of accidents and diseases. These results stress the need for early contact of veterinary students with issues related to occupational health.

Palabras clave: Medicina Veterinaria; Estudiantes ingresantes; Riesgos ocupacionales; Zoonosis

Keywords: Veterinary Medicine; Freshmen; Occupational risks; Zoonoses

La motivación predominante para la elección de una carrera es de índole expresiva y se asocia con diferentes resultados cognitivos, afectivos y conductuales (Masjuan, 2005, Orsini et al., 2016). El área de veterinaria se destaca por este tipo de motivación, con un gran interés por los animales y las ciencias médicas (Sans et al., 2011) y la frase "siempre me gustó esta profesión" es prevalente entre los estudiantes (Borlido et al., 2020). Como contrapartida, es poco probable que elijan esta disciplina quienes no se sienten atraídos por los animales, la ciencia o la medicina (Ilgen et al., 2003). No obstante, aunque los estudiantes poseen un deseo intrínseco y gran motivación para cuidar y trabajar con animales, tienen inicialmente un profundo déficit de conocimientos sobre sus comportamientos y poseen habilidades limitadas para su manejo (Dunne et al., 2018, Lilly et al., 2020).

Una de las prioridades de los programas de ciencias veterinarias es la preparación de graduados para una 
amplia gama de sectores (Feakes et al., 2019). Entre ellos, la motivación de los estudiantes es muy alta cuando las actividades involucran la práctica clínica y el trabajo con animales (Parkinson et al., 2006). El enfoque principal de la carrera es el desarrollo de habilidades clínicas para que los estudiantes alcancen las competencias básicas necesarias para convertirse en practicantes (Rösch et al., 2014). En ese camino, los accidentes ocupacionales son muy frecuentes e involucran mayoritariamente a los animales y los elementos punzo cortantes (Arce et al., 2016, de Mattos et al., 2017, Riley et al., 2016, Tarabla et al., 2019). Aproximadamente cuatro de cada seis estudiantes en Argentina han sufrido estos accidentes durante la revisión clínica de pacientes pero paradójicamente, estas actividades son percibidas como de riesgo medio o bajo por los alumnos (Tarabla et al., 2019). Por otra parte, en un futuro cercano los ingresantes van a estar expuestos a numerosas zoonosis (Sánchez et al., 2017), pero tienen un escaso conocimiento sobre las especies animales susceptibles y las formas de transmisión de estas enfermedades (Molineri et al., 2014). Es probable que las características demográficas de los estudiantes incidan sobre sus motivaciones para estudiar veterinaria y que ellos no estén preparados para enfrentar los peligros ocupacionales a los que se verán expuestos durante el cursado. Los objetivos de este trabajo fueron: a) describir los motivos para elegir la carrera de veterinaria, b) investigar las percepciones de los riesgos de sufrir accidentes y zoonosis y c) buscar posibles asociaciones con características demográficas en ingresantes a la Facultad de Ciencias Veterinarias de la Universidad Nacional del Litoral.

En marzo de 2017 se desarrolló un estudio observacional transversal basado en una encuesta anónima con cuestionario estructurado en ingresantes a la Facultad de Ciencias Veterinarias de la Universidad Nacional del Litoral (FCV UNL). El tamaño de la muestra fue de 137 estudiantes (error absoluto 5\%, nivel de confianza 95\%, frecuencia esperada $30 \%, \mathrm{~N}=340$ ). De ese total estimado, 130 ingresantes completaron las respuestas requeridas. Las percepciones de la probabilidad de zoonosis y lesiones fueron comparadas entre sí mediante correlación de Spearman. Para la búsqueda de asociaciones mediante tablas de contingencia las variables con más de dos categorías fueron transformadas en dicotómicas (motivaciones: alta/ mediabaja, residencia familiar: rural/ urbana, percepción de la probabilidad de accidentes y lesiones: alta/ mediabaja). El análisis estadístico incluyó $x 2$ y correlación de Spearman.

Los encuestados tenían $18,9 \pm 1,7$ años de edad y el $62,3 \%$ eran mujeres. Las residencias familiares estaban ubicadas en áreas urbanas $(70,9 \%)$, rurales $(15,0 \%)$ y suburbanas $(14,2 \%)$ de Santa Fe $(50,9 \%)$, Entre Ríos $(35,1 \%)$ y otras siete provincias argentinas. No se registraron asociaciones significativas entre género, edad y lugar de residencia. El $68,4 \%$ había egresado de bachilleratos; $26,2 \%$ de escuelas agrotécnicas y $5,4 \%$ de otras opciones. Las orientaciones más frecuentes fueron: biológica $(47,7 \%)$, contable $(19,2 \%)$, humanística $(16,9 \%)$ y agropecuaria $(6,7 \%)$.

Los resultados reflejan una lógica dispersión de intereses entre quienes aún no dieron sus primeros pasos en la actividad veterinaria, pero la gran mayoría se encuadró dentro del área biológica (Figura 1).

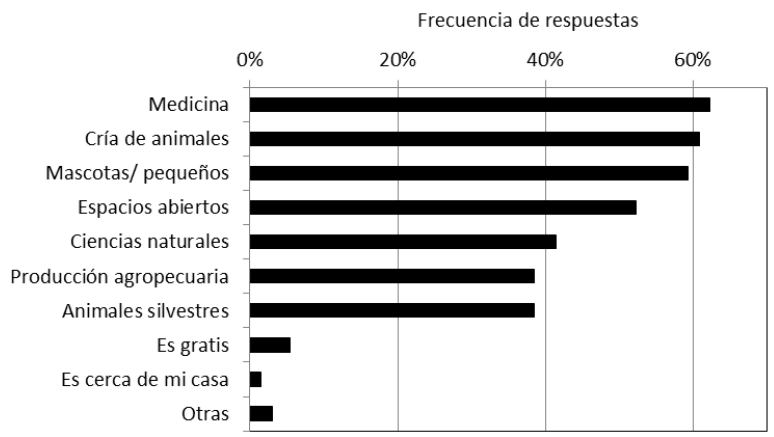

Figura 1. Motivaciones para estudiar veterinaria en alumnos ingresantes, Facultad de Ciencias Veterinarias, Universidad Nacional del Litoral $(n=130)$.

La percepción de los ingresantes a las carreras en ciencias de la salud está sustentada en la experimentación y la práctica clínica en hospitales durante el cursado y en una orientación centrada en las especialidades farmacéutica, médica o veterinaria (Masjuan, 2005). Entre los ingresantes a esta última disciplina se destaca la preponderancia de la imagen del veterinario como un "médico animal" (Sans et al., 2011). Esta preferencia por la medicina fue la más prevalente entre los encuestados. Esta respuesta no estuvo asociada con un alto interés en alguna especie en particular, con la única excepción de la fauna silvestre $(P<0,048)$. En orden de frecuencia siguieron dos respuestas genéricas ("cría de animales", "espacios abiertos") y una más específica ("mascotas/ pequeños animales").

Las intenciones de los estudiantes de veterinaria son fuertes predictores de sus comportamientos futuros (Feakes et al., 2019). Aunque puede no perdurar por largo tiempo luego de graduados (Heath, 2007), la preferencia por los grandes animales productores de alimentos está asociada a las experiencias de los estudiantes previas al ingreso a la carrera (Lenarduzzi et al., 2009). En este trabajo, el deseo de trabajar con bovinos fue más frecuente entre los varones $(P=0,005)$, mientras que las mujeres manifestaron mayor motivación para trabajar con caninos y felinos domésticos $(P<0,001)$. No hubo diferencias significativas entre géneros en las otras especies. Por su parte, la expectativa de trabajar con caninos fue mayor entre los residentes urbanos $(P=0,012)$.

Aunque no fue la más frecuente, la motivación por el trabajo rural fue mencionada por cuatro de cada 10 encuestados. Las intenciones de los ingresantes no se pueden analizar íntegramente con un único estudio 
transversal y es claramente una de las limitantes del presente trabajo. A modo de ejemplo, aunque a priori se pueda pensar en una relación directa entre la actividad agropecuaria y el trabajo en espacios abiertos, ambas motivaciones no estuvieron significativamente asociadas $(P=0,598)$. Más aún, este último sólo estuvo asociado con el interés por la fauna silvestre $(P<0,053)$. Lógicamente, la motivación por la actividad agropecuaria fue más frecuente entre quienes mostraban un alto interés por trabajar con bovinos $(P<0,001)$, ovinos $(P=0,003)$ y porcinos $(P=0,050)$. Como contrapartida, este grupo mostró una menor expectativa en trabajar con fauna silvestre $(P=0,026)$, caninos $(P=0,002)$ y felinos domésticos $(P=0,001)$. De manera similar, la cría de animales estuvo asociada positivamente con un alto interés en los bovinos $(P<0,001)$ y los equinos $(P=0,008)$ y negativamente con la fauna silvestre $(P=0,002)$, los caninos $(P=0,022)$ y los felinos domésticos $(P=0,031)$.

Aparte de la experiencia en el manejo de animales o la demografía de los estudiantes, las preferencias por determinadas especies son las que explican la mayor proporción de variación en la intención en trabajar en un sector profesional específico (Feakes et al., 2019). La disminución del número de veterinarios que trabajan con grandes animales de granja, principalmente bovinos y ovinos es motivo de preocupación en muchos países (Lenarduzzi et al., 2009). En tiempos recientes, las intenciones de los estudiantes sobre la futura práctica profesional priorizan la práctica clínica mixta o de animales de compañía y la fauna silvestre (Feakes et al., 2019, Sans et al., 2011). En la FCV UNL el perfil de formación estuvo influenciado por los cambios en los modelos de producción agropecuaria y la demanda de la sociedad que paulatinamente fue dando una mayor importancia a los animales de compañía (Galván et al., 2012). Aunque el trabajo agropecuario tuvo un número igual de menciones que la fauna silvestre, el deseo de trabajar con bovinos fue calificado como alto por una mayor proporción de encuestados. Otras especies que superaron la frecuencia del $50 \%$ fueron los caninos, seguidos por los felinos domésticos y en quinto término la fauna silvestre (Figura 2)

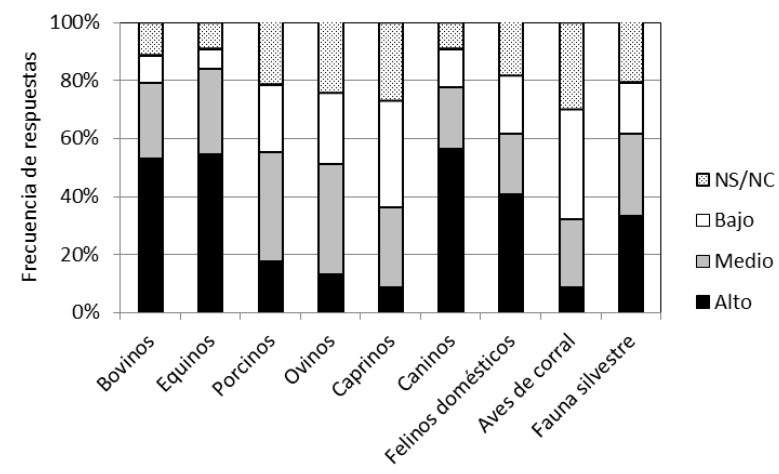

Figura 2. Interés de ingresantes a veterinaria en trabajar con una determinada especie, Facultad de Ciencias Veterinarias, Universidad Nacional del Litoral $(n=130)$.
Durante la carrera de veterinaria, los estudiantes se exponen a peligros biológicos, físicos y ergonómicos (Arce et al., 2016, de Mattos et al., 2017, Sánchez et al., 2017, Tarabla et al., 2018). Entre los ingresantes, la percepción del riesgo de sufrir enfermedades o lesiones ocupacionales durante el cursado fue mayor en el caso de las contusiones y heridas (Figura 3 ).

Las mayores frecuencias de respuestas NS/NC se registraron en las probabilidades de sufrir alergias ocupacionales o exposición solar excesiva. La prevalencia de síntomas alérgicos en los ingresantes a medicina veterinaria es similar a la de la población general (Lehnert et al., 2020), mientras que son escasas las investigaciones publicadas sobre alergias específicas por las actividades propias de la carrera Jolie et al., 1998, Samadi et al., 2012). En contraposición, la sobreexposición al sol es una de las principales causas de afecciones ocupacionales en estudiantes en Argentina (Tarabla et al., 2019).

En 778 estudiantes de 13 carreras de Veterinaria ubicadas en nueve provincias argentinas la frecuencia de diagnósticos de enfermedades zoonóticas fue del $6 \%$ (Tarabla et al., 2019), mientras que la incidencia de casos de zoonosis se eleva notablemente en los primeros años posgraduación (Molineri et al., 2013). Al inicio del cursado los estudiantes tienen un nivel de conocimiento limitado sobre los animales que pueden ser afectados y las formas que estas enfermedades pueden ser trasmitidas al ser humano (Molineri et al., 2014). Las enfermedades más mencionadas por los encuestados fueron: rabia $(43,1 \%)$, tuberculosis $(23,8 \%)$, brucelosis $(18,5 \%)$, toxoplasmosis $(15,4 \%)$, carbunclo $(16,2 \%)$, leptospirosis $(14,6 \%)$, sarna $(13,8 \%)$ y triquinosis $(13,1 \%)$. Es interesante notar que las respuestas "No sabe/no contesta" (NS/NC) tuvieron un registro del $36,9 \%$ y las respuestas erróneas o no específicas del $22,3 \%$. Esto puede avalar la propuesta de introducir de manera temprana a los ingresantes en las características generales de las enfermedades transmitidas de los animales al hombre. En el caso de las percepciones de los estudiantes sobre el riesgo de contraer una zoonosis de una especie animal determinada, las respuestas NS/NC no estuvieron asociadas al género, a la predisposición para trabajar con una determinada especie (alta/ media- baja) ni a la residencia familiar, con la sola excepción del ganado bovino. En esta especie, NS/NC fue más frecuente entre quienes tenían un interés bajo o medio para trabajar en esta práctica $(P=0,023)$ y quienes residían en ciudades $(P=0,038)$. Otras variables no medidas en este estudio, como la situación sanitaria regional o nacional pudieron también influenciar estos resultados.

La mayoría de los encuestados estimaron como medio o alto el riesgo que los porcinos, los bovinos y las mascotas (caninos y felinos) les transmitan una zoonosis. Por su parte las frecuencias de NS/NC fueron $\geq$ al $40 \%$ en los casos de los caprinos, los ovinos, los equinos y la fauna silvestre (Figura 4). 


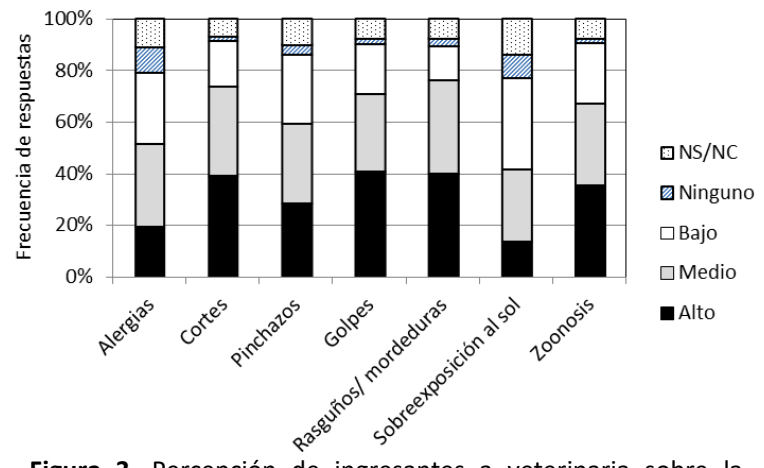

Figura 3. Percepción de ingresantes a veterinaria sobre la probabilidad de sufrir enfermedades profesionales o accidentes ocupacionales durante la carrera, Facultad de Ciencias Veterinarias, Universidad Nacional del Litoral $(n=130)$.

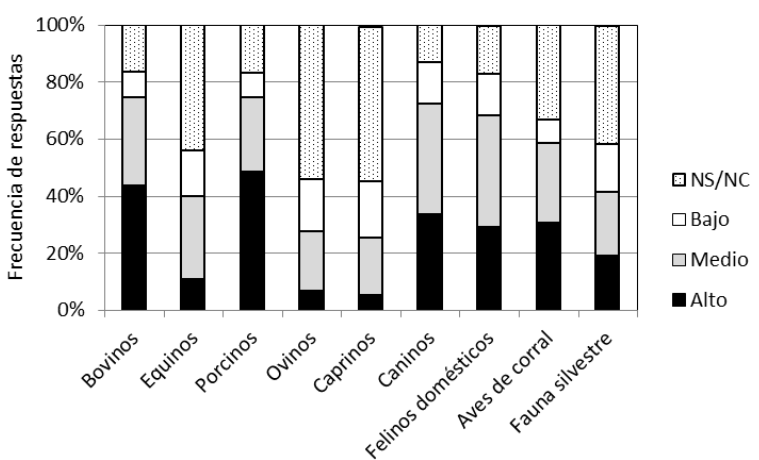

Figura 4. Percepción de ingresantes a veterinaria sobre la probabilidad que una especie animal le transmita una zoonosis durante la carrera, Facultad de Ciencias Veterinarias, Universidad Nacional del Litoral $(n=130)$.

En Argentina, los animales que más frecuentemente estuvieron involucradas en accidentes con estudiantes de veterinaria fueron los bovinos, los caninos y los felinos domésticos y en cuarto orden, los equinos con un $46,41,22$ y $21 \%$ de estudiantes lesionados (Tarabla et al., 2019). En el presente trabajo, sin embargo, fue la equina la especie considerada como de mayor riesgo de ocasionar lesiones (Figura 5).

La mayoría de los accidentes de los estudiantes con equinos suelen ocurrir durante prácticas desarrolladas fuera de las instalaciones universitarias (Riley et al., 2015) y, como en otras especies, está influenciada no sólo por la agresividad de la especie y la habilidad para su manejo por parte de los profesionales y estudiantes, sino por la probabilidad y el tiempo de exposición. Esto tendrá obvias variaciones en cada unidad académica.

Las frecuencias de NS/NC fueron $\geq 40 \%$ en el caso de la percepción de la probabilidad de accidentes con ovinos, aves de corral, caprinos y fauna silvestre. Estas respuestas no estuvieron asociadas al género, a la predisposición para trabajar con una determinada especie (alta/ media-baja) ni a la residencia familiar.

La percepción del riesgo de adquirir una zoonosis o sufrir una lesión en el trabajo con una determinada especie animal estuvieron positivamente correlacionadas entre sí: bovinos $(r=0,37 ; P<0,001)$, ovinos ( $r=$
$0,40 ; P=0,009)$, caprinos $(r=0,56 ; P<0,001)$, porcinos $(\mathrm{r}=0,31 ; P=0,006)$, caninos $(\mathrm{r}=0,64 ; P<0,001)$, felinos $(r=0,49 ; P<0,001)$, aves de corral $(r=0,32 ; P=0,026)$, fauna silvestre $(r=0,33 ; P=0,010)$, con la única excepción de los equinos ( $r=0,06 ; P<0,631)$ que registró una percepción de riesgos más alta para la probabilidad de lesiones que para la de zoonosis.

Los elementos punzocortantes, los animales y los químicos fueron considerados los factores con mayor probabilidad de estar involucrados en lesiones o enfermedades (Figura 6).

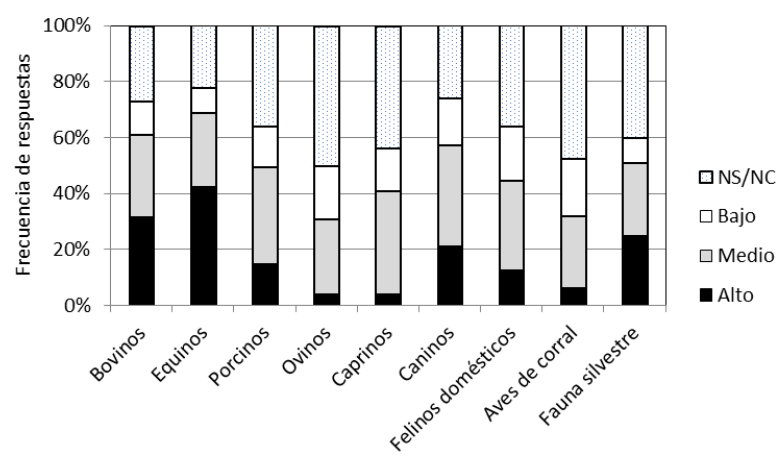

Figura 5. Percepción de ingresantes a veterinaria sobre la probabilidad que una especie animal le ocasione lesiones durante la carrera, Facultad de Ciencias Veterinarias, Universidad Nacional del Litoral $(n=130)$.

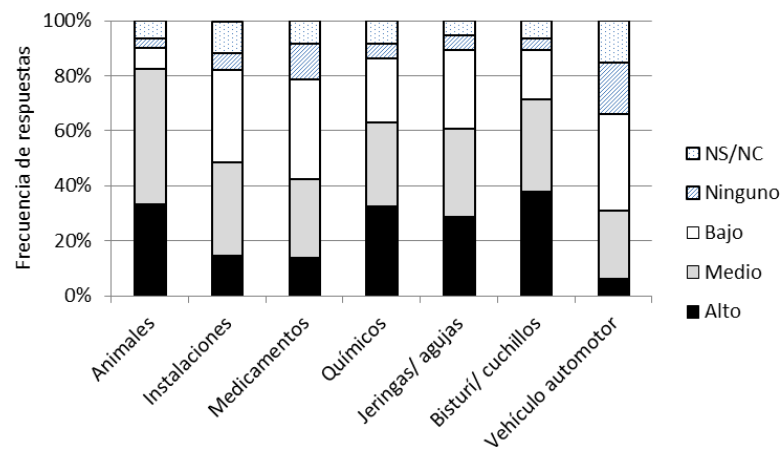

Figura 6. Percepción de ingresantes a veterinaria sobre la probabilidad que un animal o un elemento le ocasione lesiones o enfermedades durante la carrera, Facultad de Ciencias Veterinarias, Universidad Nacional del Litoral $(n=130)$.

La percepción de la probabilidad de accidentes con químicos tuvo un registro importante. La exposición de los estudiantes a formaldehidos, gases anestésicos y drogas antineoplásicas ha sido comunicada en trabajos recientes (Arce et al., 2016). No obstante, la probabilidad de lesiones o enfermedades agudas es baja (Tarabla et al., 2019). Los encuestados estimaron que la probabilidad de accidentes ocupacionales era mayor en el campo (alta $36,2 \%$, media $39,2 \%$, baja $13,8 \%$, ninguna $5,4 \%, \mathrm{NS} / \mathrm{NC} 5,4 \%$ ) que en el consultorio (alta $10,0 \%$, media $41,5 \%$, baja $33,1 \%$, ninguna $6,9 \%$, NS/NC $8,5 \%$ ) o en el tránsito in itinere (alta $7,7 \%$, media $32,3 \%$, baja $37,7 \%$, ninguna $13,8 \%$, NS/NC $8,5 \%$ ). Estas percepciones contradicen la probabilidad de lesiones causadas en la práctica diaria que es muy alta, tanto en la práctica rural como en la atención de mascotas en 
consultorios (Tarabla, 2017). La posibilidad de accidentes durante el traslado por razones laborales tuvo el registro más bajo, coincidiendo con su baja frecuencia en estudiantes (Tarabla et al., 2019). Esta probabilidad, no obstante, sube drásticamente entre los profesionales veterinarios en nuestro país, cuando aumenta la probabilidad y el tiempo de exposición al tránsito vehicular por razones laborales (Huertas et al., 2019, Molineri et al., 2016).

Las respuestas NS/NC fueron frecuentes en el reconocimiento de algunas zoonosis y en las percepciones de los riesgos de sufrir accidentes o contraer enfermedades zoonóticas durante la carrera. La diversidad de procedencias y motivaciones, unidas a la dispersa percepción sobre la probabilidad de accidentes ocupacionales o enfermedades profesionales reafirman la necesidad de un contacto precoz de los estudiantes de veterinaria con temas relacionados con la salud ocupacional.

\section{Agradecimientos.}

A los integrantes de Introducción a la Veterinaria, Ciclo Básico, FCV UNL, especialmente a los alumnos adscriptos E. Picard y A. Battú por la colaboración prestada. Al Dr. M. L. Signorini por la lectura crítica de la presente comunicación.

\section{Referencias}

Arce C, León D, Breña M, Falcón N. 2016. Accidentes y lesiones en estudiantes de Medicina Veterinaria y Zootecnia en una Universidad de Lima -Perú. Salud Tecnol. Vet. 4: 1-8.

Borlido C, Lujambio V, González S, Porro A, Passarini J. 2020. ¿Qué sabemos de los estudiantes que ingresan a la carrera de veterinaria en Uruguay? Rev. Argent. Educ. Superior 12: 78-96.

de Mattos Brose M, Silva de Assis MC. 2017. Acidentes de trabalho em um Hospital Veterinário Universitário: estudo de prevalencia. Conexão Ciênc. (UNIFOR) 12: 13-21.

Dunne K, Brereton B, Duggan V, Campion D. 2018. Motivation and prior animal experience of newly enrolled veterinary nursing students at two Irish third-level institutions. J. Vet. Med. Educ. 45: 413-422.

Feakes AM, Palmer EJ, Petrovski KR, Thomsen DA, Hyams JH, Cake MA, Webster B, Barber SR. 2019. Predicting career sector intent and the theory of planned behaviour: survey findings from Australian veterinary science students. BMC Vet. Res. 15: 27.

Galván SM, Sacco Hümöller S. 2012. Análisis comparativo de las trayectorias profesionales de los graduados de Medicina Veterinaria (FCV-UNL) XIII Jorn. Divulgación Téc. Cien. FCV UNR, Casilda, Santa Fe, 2 pp.

Gómez de la Torre N, Tarabla HD. 2015. Accidentes laborales, enfermedades profesionales y uso de elementos de protección personal en veterinarios de pequeños animales. InVet UBA 17: 23-27.
Heath TJ. 2007. Longitudinal study of veterinary students and veterinarians: effects of growing up on a farm with animals. Aust. Vet. J. 85: 296-299.

Huertas PS, Molineri Al, León EA, Signorini ML, Tarabla HD. 2019. Factores asociados a accidentes in itinere en veterinarios de grandes animales. Rev. Cien. Ab Intus UNRC 3: 71-76.

Ilgen DR, Lloyd JW, Morgeson FP, Johnson MD, Meyer CJ, Marrinan M. 2003. Personal characteristics, knowledge of the veterinary profession, and influences on career choice among students in the veterinary school applicant pool. J. Am. Vet. Med. Assoc. 223: 1587-1594.

Jolie R, Backstrom L, Thomas C. 1998. Health problems in veterinary students after visiting a commercial swine farm. Can. J. Vet. Res. 62: 44-48.

Lehnert M, Beine A, Hoffmeyer F, Taeger D, Brüning T, Raulf M. 2020. Self-reported survey on allergy symptoms among firstyear students in Veterinary Medicine: a preamble to the AllergoVet Cohort Study. Adv. Exp. Med. Biol. 1279: 9-14.

Lenarduzzi R, Sheppard GA, Slater MR. 2009. Factors influencing the choice of a career in food-animal practice among recent graduates and current students of Texas A\&M University, College of Veterinary Medicine. J. Vet. Med. Educ. 36: 7-15.

Lilly ML, Gonçalves Arruda A, Proudfoot KL, Herron ME. 2020. Evaluation of companion animal behavior knowledge among first-year veterinary students before and after an introductory animal behavior course. J. Am. Vet. Med. Assoc. 256: 11531163.

Masjuan JM. 2005. Progresos en los aprendizajes, características de los estudios y motivaciones de los estudiantes. Papers Rev. Sociol. Univ. Auton. Barcelona 76: 97133.

Molineri Al, Signorini ML, Pérez L, Tarabla HD. 2013. Zoonoses in rural veterinarians in the central area of Argentina. Aust. J. Rur. HIth. 21: 285-290.

Molineri Al, Signorini ML, Ruiz M, Marenghi M, Tarabla HD. 2014. Awareness of zoonoses in freshmen entering the Veterinary School. Braz. J. Vet. Sci. 21: 239-242.

Molineri Al, Signorini ML, Tarabla HD. 2016. Distribución espacial de accidentes de tránsito, percepción de riesgos y uso de elementos de protección personal in itinere en veterinarios de grandes animales en la Provincia de Santa Fe. Analecta Vet. UNLP 36: 23-27.

Orsini C, Binnie VI, Wilson SL. 2016. Determinants and outcomes of motivation in health professions education: a systematic review based on self-determination theory. J. Educ. Eval. Health Prof. 13: 19.

Parkinson TJ, Gilling M, Suddaby GT. 2006. Workload, study methods, and motivation of students within a BVSc program. J. Vet. Med. Educ. 33: 253-265.

Riley CB, Liddiard JR, Thompson K. 2015. A cross-sectional study of horse-related injuries in Veterinary and Animal Science students at an Australian University. Animals 5: 951964. 
Riley CB, McCallum S, MacDonald JA, Hill KE. 2016. A prospective observational study of needle-handling practices at a University Veterinary Teaching Hospital. N. Z. Vet. J. 64: 117-120.

Rösch T, Schaper E, Tipold A, Fischer MR, Dilly M, Ehlers JP. 2014. Clinical skills of veterinary students - a cross-sectional study of the self-concept and exposure to skills training in Hannover, Germany. BMC Vet. Res. 10: 969.

Samadi S, Spithoven J, Jamshidifard AR, Berends BR, Lipman L, Heederik DJJ, Wouters IM. 2012. Allergy among veterinary medicine students in The Netherlands. Occup. Environ. Med. 69: 48-55.

Sánchez A, Prats-van der Ham M, Tatay-Dualde J, Paterna A, de la Fe C, Gómez-Martín Á, Corrales JC, Contreras A. 2017. Zoonoses in veterinary students: a systematic review of the literature. PLoS One 12: e0169534.

Sans P, Mounier L, Bénet JJ, Lijour B. 2011. The motivations and practice-area interests of first-year French veterinary students (2005-2008). J. Vet. Med. Educ. 38: 199-207.

Tarabla HD. 2017. Riesgos laborales en Medicina Veterinaria en América Latina y el Caribe. Revisión. Rev. Cien. Vet. (Costa Rica) 35: 65-84.

Tarabla HD, Molineri Al, Robin H, Signorini ML. 2019. Riesgos ocupacionales en estudiantes de Veterinaria en Argentina. Rev. Vet. UNNE 30: 83-67. 